\title{
Effects of stressor controllability on psychophysiological, cognitive and behavioural responses in patients with major depression and dysthymia
}

\author{
C. Diener ${ }^{1 * \dagger} \uparrow$, C. Kuehner ${ }^{2} \uparrow$, W. Brusniak ${ }^{1}$, M. Struve ${ }^{1}$ and H. Flor ${ }^{1}$ \\ ${ }^{1}$ Department of Cognitive and Clinical Neuroscience, Central Institute of Mental Health, University of Heidelberg, Mannheim, Germany \\ ${ }^{2}$ Research Group Longitudinal and Intervention Research, Department of Psychiatry and Psychotherapy, Central Institute of Mental Health, \\ Mannheim, Germany
}

Background. The experience of uncontrollability and helplessness in the face of stressful life events is regarded as an important determinant in the development and maintenance of depression. The inability to successfully deal with stressors might be linked to dysfunctional prefrontal functioning. We assessed cognitive, behavioural and physiological effects of stressor uncontrollability in depressed and healthy individuals. In addition, relationships between altered cortical processing and cognitive vulnerability traits of depression were analysed.

Method. A total of 26 unmedicated depressed patients and 24 matched healthy controls were tested in an expanded forewarned reaction (S1-S2) paradigm. In a factorial design, stressor controllability varied across three consecutive conditions: (a) control, $(b)$ loss of control and $(c)$ restitution of control. Throughout the experiment, error rates, ratings of controllability, arousal, emotional valence and helplessness were assessed together with the post-imperative negative variation (PINV) of the electroencephalogram.

Results. Depressed participants showed an enhanced frontal PINV as an electrophysiological index of altered information processing during both loss of control and restitution of control. They also felt more helpless than controls. Furthermore, frontal PINV magnitudes were associated with habitual rumination in the depressed subsample.

Conclusions. These findings indicate that depressed patients are more susceptible to stressor uncontrollability than healthy subjects. Moreover, the experience of uncontrollability seems to bias subsequent information processing in a situation where control is objectively re-established. Alterations in prefrontal functioning appear to contribute to this vulnerability and are also linked to trait markers of depression.

Received 22 June 2007; Revised 20 March 2008; Accepted 27 March 2008; First published online 9 May 2008

Key words: Depression, learned helplessness, post-imperative negative variation, rumination, stress.

\section{Introduction}

Human as well as animal studies emphasize the maladaptive role of stress exposure in the development of depression (Czéh et al. 2001; Alfonso et al. 2005; Henn \& Vollmayr, 2005). There is evidence that the degree of behavioural control modulates the impact of environmental stressors (Maier, 1984). Consequences of stressor uncontrollability in humans range from cognitive, motivational and emotional alterations (Maier \& Seligman, 1976) to neuroendocrinological, neurochemical and functional as well as

\footnotetext{
* Address for correspondence: C. Diener, Ph.D., Department of Cognitive and Clinical Neuroscience, Central Institute of Mental Health, Square J5, 68159 Mannheim, Germany.

(Email : carsten.diener@zi-mannheim.de)

† These authors contributed equally to this work.
}

structural brain changes (Breier, 1989) that are in line with core features of depression. Despite these parallels, studies investigating the psychobiological effects of stressor uncontrollability in depressed individuals are rare.

Neuroimaging studies of depression point to dysfunctions in the limbic-thalamic-cortical network, indicating hypoactivity in prefrontal brain areas including the cingulate gyrus during resting states (Mayberg, 1997; Pizzagalli et al. 2005). In contrast, a majority of studies examining activation during cognitively demanding tasks identified prefrontal hyperactivation in depressed patients at similar performance levels as controls (Harvey et al. 2005; Wagner et al. 2006; Matsuo et al. 2007), potentially indicating compensatory activation in order to maintain adequate levels of performance. 
Slow cortical potential studies have shown that the post-imperative negative variation (PINV) is a suitable indicator of information processing during uncontrollability over aversive events. In healthy subjects, enhanced PINV magnitudes over frontal recording sites have been found during an unexpected change from an escape paradigm to uncontrollability (Rockstroh et al. 1979; Elbert et al. 1982) and during unpredictable response outcome contingencies in general (Kathmann et al. 1990). In addition, it has been shown that anhedonic individuals display higher PINV magnitudes during uncontrollability (Lutzenberger et al. 1981). In depressed patients, Bolz \& Giedke (1981) found higher PINV magnitudes when an aversive stimulus was uncontrollable or when control was restricted. These data suggest that the PINV depicts a frontal response evaluation in situations of stressor uncontrollability or uncertainty about the appropriate response. Under such conditions, depressed patients seem to be more vulnerable than healthy controls (Bolz \& Giedke, 1981; Breier, 1989).

In the present study, we expanded a standard forewarned reaction (S1-S2) paradigm (Rockstroh et al. 1979) to assess cognitive, behavioural and physiological effects of stressor uncontrollability in depressed and healthy individuals. Stressor controllability varied across three consecutive conditions: (a) control, (b) loss of control and (c) restitution of control. We hypothesized that depressed participants would be more prone to uncontrollability as indexed by enhanced frontal PINV magnitudes and pronounced feelings of helplessness and uncontrollability. Additionally, based on learned helplessness theory (Maier \& Seligman, 1976), we expected that loss of control would bias subsequent information processing particularly in depressed subjects, as indicated by a pattern of elevated frontal PINV magnitude, prolonged feelings of helplessness and uncontrollability, and increased error rates during restitution of control. In contrast, we did not expect group differences in parietal PINV magnitudes during loss of control and restitution of control. Finally, we hypothesized that a larger frontal PINV - particularly under restitution of control-would be associated with higher levels of depression and with habitual rumination, the latter describing a cognitive vulnerability trait to depression characterized by perpetual negative self-focusing (Kuehner et al. 2007a).

\section{Method}

\section{Participants}

Twenty-six medication-free depressed patients with a diagnosis of major depressive disorder $(n=19)$ or dysthymia $(n=7)$ in the age range of $18-60$ years were recruited from the Department of Psychiatry and Psychotherapy, Central Institute of Mental Health, Mannheim, Germany $(n=10)$ and by public announcements $(n=16)$. Twenty-four age-, educationand gender-matched healthy controls were recruited by advertisement from the local community.

Participants were examined using the structured clinical interview for Diagnostic and Statistical Manual of Mental Disorders (DSM-IV) (APA, 1994) Axis I disorders (First et al. 1997). Healthy participants were excluded if they met criteria for a current DSM-IV Axis I disorder or lifetime criteria for any affective disorder. General exclusion criteria were current alcohol or drug abuse, current use of psychotropic medication and current or lifetime psychotic symptoms and neurological problems. A co-morbid anxiety disorder was not an exclusion criterion in the depressed sample.

Participants completed the Beck Depression Inventory II (BDI-II; Beck et al. 1996) and were evaluated for depression severity using the Hamilton Rating Scale for Depression (HAMD; Hamilton, 1960). Psychosocial functioning was rated using the social and occupational functioning assessment scale (APA, 1994). Habitual rumination was assessed by the German version of the response styles questionnaire (Nolen-Hoeksema \& Morrow, 1991; Kuehner et al. $2007 b$ ) with the subscales symptom-focused and selffocused rumination. A ruminative coping style in response to depressed mood has been found to exaggerate and prolong dysphoric episodes (NolenHoeksema, 2004). The study was in concordance with the declaration of Helsinki and was approved by the Medical Ethics Committee of the Medical Faculty Mannheim at the University of Heidelberg, Germany. After complete description of the study to the subjects, written informed consent was obtained.

Basic demographic and clinical sample characteristics are provided in Table 1. A large percentage of depressed patients $(73.9 \%)$ had suffered from previous episodes of depression.

\section{Experimental design}

The experiment consisted of a modified forewarned (S1-S2) reaction paradigm. S1 was a $600 \mathrm{~Hz} / 60 \mathrm{~dB}$ tone of $4 \mathrm{~s}$ duration immediately followed by $\mathrm{S} 2$, which was a $1000 \mathrm{~Hz} / 60 \mathrm{~dB}$ tone of $1 \mathrm{~s}$ duration. In case of aversive stimulation, an electrical stimulus of $1 \mathrm{~ms}$ duration was applied to the index finger of the non-dominant hand following S2 termination. The intertrial interval varied randomly between $5 \mathrm{~s}$ and $9 \mathrm{~s}$ (mean $=7 \mathrm{~s})$. Subjects were instructed to respond to $\mathrm{S} 2$ onset by pressing the correct (left $v$. 
Table 1. Sample characteristics

\begin{tabular}{|c|c|c|c|c|}
\hline Variable & $\begin{array}{l}\text { Depressed } \\
\text { subjects } \\
(n=26)\end{array}$ & $\begin{array}{l}\text { Healthy } \\
\text { subjects } \\
(n=24)\end{array}$ & $\begin{array}{l}F(1,48) \\
\text { or } \chi^{2}(1)\end{array}$ & $p$ \\
\hline Age (years) & $47.19(8.94)$ & $44.75(9.02)$ & 0.92 & N.S. \\
\hline Gender ( $\%$ female) & 54.16 & 64.71 & 0.46 & N.S. \\
\hline Length of education (years) & $11.00(1.57)$ & $11.13(1.48)$ & 0.08 & N.S. \\
\hline \multicolumn{5}{|l|}{ DSM-IV diagnosis (SCID) } \\
\hline Major depression (\%) & 73.08 & - & & \\
\hline Pure dysthymia (\%) & 26.92 & - & & \\
\hline Co-morbid anxiety diagnosis $(n)$ & 2 & - & & \\
\hline Age at onset (years) & $30.42(22.03)$ & - & & \\
\hline No. of episodes & $3.32(3.17)$ & - & & \\
\hline HAMD depression score & $18.69(6.66)$ & $0.25(0.44)$ & 182.89 & $<0.001$ \\
\hline BDI-II depression score & $25.24(10.62)$ & $0.78(1.86)$ & 123.48 & $<0.001$ \\
\hline Psychosocial functioning (SOFAS) & $61.35(10.48)$ & $99.58(2.04)$ & 308.21 & $<0.001$ \\
\hline Electrical stimulus amperage $(\mathrm{mA})$ & $3.34(2.39)$ & $4.13(3.79)$ & 0.88 & N.s. \\
\hline
\end{tabular}

Values are given as mean (standard deviation), \% or $n$.

N.s., Non-significant; DSM-IV, Diagnostic and Statistical Manual of Mental Disorders, 4th edition (APA, 1994); SCID, Structured Clinical Interview for DSM-IV Axis I disorders (First et al. 1997); HAMD, Hamilton Rating Scale for Depression (Hamilton, 1960); BDI-II, Beck Depression Inventory, 2nd revision (Beck et al. 1996); SOFAS, Social and Occupational Functioning Scale (APA, 1994).

right) button in order to avoid aversive electrical stimulation.

Controllability of the aversive stimulus varied across three successive conditions of 40 trials each. In the first condition of controllability, the aversive stimulus could be avoided by pressing either the right or the left button (counterbalanced across subjects). Without prior warning, subjects randomly received the electrical stimulus in half of the trials during the following condition of uncontrollability so that no response outcome contingency could be established. In the third condition, control was re-established, again without prior warning. The left $v$. right button press as effective response was switched between the conditions of controllability and restitution of control.

Prior to the experimental manipulation (baseline) and every 15th and 35th trial in each condition, participants rated their current arousal and emotional valence via a self-assessment manikin (Lang, 1980). Controllability and helplessness were rated on visual analogue scales. The ratings were presented on a monitor $\sim 1.2 \mathrm{~m}$ in front of the subjects.

During the experiment, the participants sat in a reclining chair in an electrically shielded room. S1 and S2 tones were presented by means of foam ear inserts (MedCaT, Erica, The Netherlands). The intensity of the pain stimulus was individually calibrated as aversive but tolerable (see Table 1). During the experiment participants had to focus a fixation-cross in the centre of the monitor to avoid eye-movement artefacts. The entire protocol lasted about $1 \mathrm{~h}$. The diagnostic interview was carried out in a separate session lasting up to $2.5 \mathrm{~h}$.

\section{Apparatus and recording technique}

Participants' scalp electroencephalography (EEG) was recorded from 30 sites based on the extended 10-20 system (Nuwer et al. 1998) referenced to linked mastoids and digitally sampled at $500 \mathrm{~Hz}$ using a Neuroscan Synamps DC amplifier (NeuroScan Inc., Herndon, VA, USA). An Easy Cap (FMS GmbH, Munich, Germany) with AgAgCl-electrodes of $10 \mathrm{~mm}$ diameter was used. The electrodes were prepared with abrasive paste (Abralyt 2000; FMS GmbH) and filled with electrode jelly (Electro-Cap International, Inc., Eaton, OH, USA). Impedances were kept below $5 \mathrm{k} \Omega$. The electro-oculogram (EOG) was recorded from tin electrodes of $9 \mathrm{~mm}$ diameter which were affixed $1 \mathrm{~cm}$ above and below the left eye (vertical EOG), and at the outer canthi (horizontal EOG) of both eyes (Picton et al. 1995).

For electrical stimulation, a constant current stimulator (model DS7A; Digitimer Ltd, Welwyn Garden City, Herts, UK) was used with custom-designed foil electrodes, which were attached to the distal and 
middle phalanges of the index finger of the nondominant hand.

\section{Data reduction and analysis}

Subjects' button presses were continuously recorded and classified as erroneous if the response was followed by electrical stimulation in the control and restitution of control conditions. Controllability and helplessness ratings were scored from 0 to 100, those for arousal and valence from 1 to 9 (Lang, 1980). The mean of the two ratings (at trial 15 and 35) per condition was subjected to the statistical analysis.

Brain vision analyzer (version 1.05; Brain Products $\mathrm{GmbH}$, Gilching, Germany) was employed for the analyses of EEG data. Based on other studies examining the PINV (Rockstroh et al. 1979; Elbert et al. 1982) and our study hypotheses, midline frontal (Fz) and parietal $(\mathrm{Pz})$ recording sites, the latter serving as a control site, were included in the analyses. Data were segmented into epochs of $9 \mathrm{~s}$ corresponding to S1 and S2 presentation ( $5 \mathrm{~s}$ ) and a $4 \mathrm{~s}$ post-imperative interval. Each segment was corrected for eye movements (Gratton et al. 1983) and semi-automatically rejected when corrupted by artifacts (i.e. voltage step/sampling point $\geqslant 50 \mu \mathrm{V}$, minimal amplitude $<-100 \mu \mathrm{V}$, maximal amplitude $>100 \mu \mathrm{V})$. The remaining trials (minimum 34 ; i.e. $85 \%$ ) were then averaged. There were no group $[F(1,48)=0.15$, N.s.], condition $[F(2,47)=0.4$, N.s. $]$ or group $\times$ condition $[F(2,47)=0.6$, N.s.] effects with regard to trials included. PINV magnitudes were defined as mean activity $(\mu \mathrm{V})$ during the segment between $800 \mathrm{~ms}$ and $3500 \mathrm{~ms}$ following S2 termination relative to a 1000 ms pre-trial baseline (see Rockstroh et al. 1979; Bolz \& Giedke, 1981; Elbert et al. 1982; Kathmann et al. 1990).

\section{Statistical analyses}

Univariate group comparisons for sociodemographic and clinical data were performed using analyses of variance (ANOVAs) for continuous and $\chi^{2}$ tests for dichotomous variables. The dependent variables were subjected to repeated-measures ANOVAs with group (depressed $v$. healthy subjects) as between- and condition (control, loss of control, and restitution of control) as within-subject factors. To test for significance, we used Pillai's trace statistic, which is most robust against violations of assumptions. In case of significant main or interaction effects, post-hoc paired comparisons were performed by means of paired-samples $t$ tests with two-tailed levels of significance.

Since female gender is regarded as an important risk factor for depression, and learned helplessness is thought to be particularly connected to the female gender role (Kuehner, 2003), we controlled for potential gender differences in all analyses. Within the patient and healthy control samples, male and female subjects did not differ with regard to any of the assessed variables presented in Table 1. Furthermore, the inclusion of gender in the analyses described below did not result in any significant main or interaction effect of this variable and did not affect the significance levels of the other variables in the respective models. Therefore, we only present results with data collapsed across gender.

Partial correlations with two-tailed significance levels are reported for the associations between frontal PINV magnitudes and measures of depression, helplessness and habitual rumination for the depressed and the healthy subsample. In these analyses, we controlled for PINV magnitudes during the initial condition of control in analyses involving the PINV under loss of control and restitution of control. In analyses involving the rumination subscales, we further adjusted for current Hamilton depression scores to assess net associations between PINV scores and cognitive vulnerability not attributable to interindividual differences in depression levels.

\section{Results \\ Rating data \\ Controllability}

For the controllability ratings a main effect of condition [Pillai's trace $F(2,47)=16.86, p<0.001$ ] was found that mirrored exactly the experimental design (Fig. 1a). Depressed and healthy subjects showed a decrease from control $($ mean $=63.40$, S.D. $=35.79)$ to loss of control $($ mean $=34.10$, S.D. $=30.00) \quad[t(49)=$ $-5.90, p<0.001$ ], followed by a subsequent increase during restitution of control $($ mean $=52.00$, S.D. $=31.82)$ $[t(49)=4.31, p<0.001]$. There were no significant group $[F(1,48)=0.204$, N.s.] or interaction effects $[F(2,47)=0.019$, N.s. $]$, indicating that the two groups did not differ in the overall amount of perceived control or across conditions (see Fig. 1a).

\section{Helplessness}

For the helplessness ratings a main effect of group $[F(1,48)=7.38, p=0.009]$ reflected elevated levels of perceived helplessness in the depressed participants $($ mean $=33.71$, S.D. $=25.80)$ compared with healthy controls $($ mean $=13.89$, S.D. $=25.82)$ throughout the experiment. There was no significant main effect for condition $[F(2,47)=1.95$, N.s.] but a marginally significant condition $\times$ group interaction $[F(2,47)=3.01$, $p=0.059]$. Depressed patients $[t(25)=0.65$, N.s.] and 

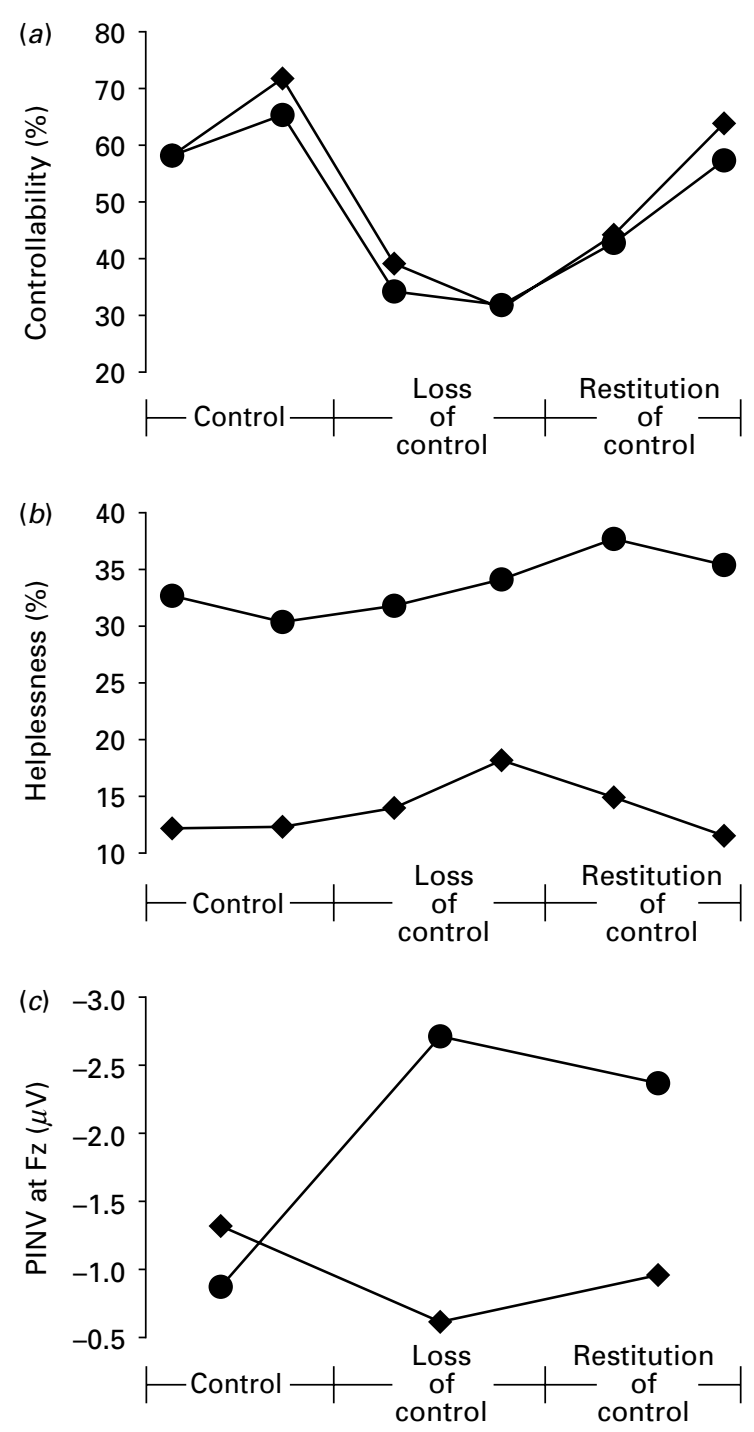

Fig. 1. Ratings of perceived (a) controllability, $(b)$ helplessness and $(c)$ magnitudes $(\mu \mathrm{V})$ of the post-imperative negative variation (PINV) at the midline frontal recording site $(\mathrm{Fz})$ during the experimental protocol for depressed $(\bullet, n=26)$ and healthy $(\bullet, n=24)$ subjects.

controls $[t(23)=1.74, \quad$ N.s. $]$ showed similar nonsignificant increases from control to loss of control $[t(49)=1.67$, N.s. $]$. However, in depressed participants the feelings of helplessness further increased during restitution of control $($ mean $=36.54$, S.D. $=30.26)$ as compared with initial control $($ mean $=31.54$, S.D. $=$ 29.69) $[t(25)=2.17, p=0.040]$, a pattern not seen in healthy participants $[t(23)=0.55$, N.s., see Fig. $1 b]$.

\section{Arousal}

The arousal ratings of the depressed participants were significantly higher throughout the experiment (mean $=0.17$, s.D. $=1.68$ v. mean $=2.66$, s.D. $=$ 1.72) [group: $F(1,48)=9.78, p=0.003$ ]. A significant condition effect $[F(2,48)=-4.94, p=0.011]$ indicated a decline in arousal from controllability (mean $=3.48$, S.D. $=3.62)$ to restitution of control (mean=3.23, S.D. $=1.91)[t(49)=-2.18, p=0.034]$. A non-significant interaction term $[F(2,47)=1.19$, N.s. $]$ indicated similar decreases in both groups.

\section{Emotional valence}

In general, depressed participants (mean $=4.87$, s.D. $=$ 1.63) rated the situation as significantly more unpleasant than healthy controls $($ mean $=3.39$, s.D. $=1.67)$ [group: $F(1,48)=10.20, p=0.002$ ]. There was neither a significant main effect for condition $[F(2,47)=2.33$, N.S.] nor a significant condition $\times$ group interaction $[F(2,47)=0.41$, N.s. $]$.

\section{Behavioural data}

\section{Errors and reaction times}

The ANOVAs for erroneous responses (ER) and for reaction times (RT) resulted in no significant effects [condition: $\operatorname{ER~} F(1,48)=2.73$, N.s. ; RT $F(2,47)=$ 0.278 ; group: ER $F(1,48)=1.35$, N.s. ; RT $F(1,48)=0.01$, N.s.; interaction: ER $F(1,48)=0.26$, N.s. ; RT $F(1,48)=$ 0.26 , N.s.].

\section{PINV}

Due to insufficient statistical power, the omnibus ANOVA resulted in no significant interaction of group, condition and electrode site $[F(2,45)=1.56$, $p=0.221]$. Therefore and according to our hypotheses, we separately report the results for the frontal and parietal recording sites.

\section{Frontal recording site}

For Fz a main effect for group $[F(1,48)=5.88, p=0.019]$ was found. Overall, depressed participants showed higher PINVs (mean $=-1.98$, s.D. $=1.49$ ) than healthy controls $\quad($ mean $=-0.97$, S.D. $=1.49)$. Furthermore, we identified a marginally significant main effect for condition $[F(2,47)=2.98, p=0.060]$ and a highly significant condition $\times$ group interaction $[F(2,47)=$ 8.54, $p<0.001]$. Within-group analyses revealed that in contrast to healthy controls $[t(23)=-1.07$, N.s.] depressed participants responded with an enhanced PINV $[t(25)=-3.35, p=0.003]$ during loss of control $($ mean $=-2.71$, S.D. $=2.48) \quad$ compared with initial control (mean $=-0.87$, s.D. $=1.43$ ). Additionally, depressed participants showed a higher PINV during restitution of control (mean $=-2.38$, s.D. $=1.58)$ compared with initial control $[t(25)=-4.30, p<0.001]$, a pattern not seen in healthy controls $[t(23)=-0.55$, N.s., see Fig. 1c]. 
(a) Depressed subjects $(n=26)$

Healthy subjects $(n=24)$
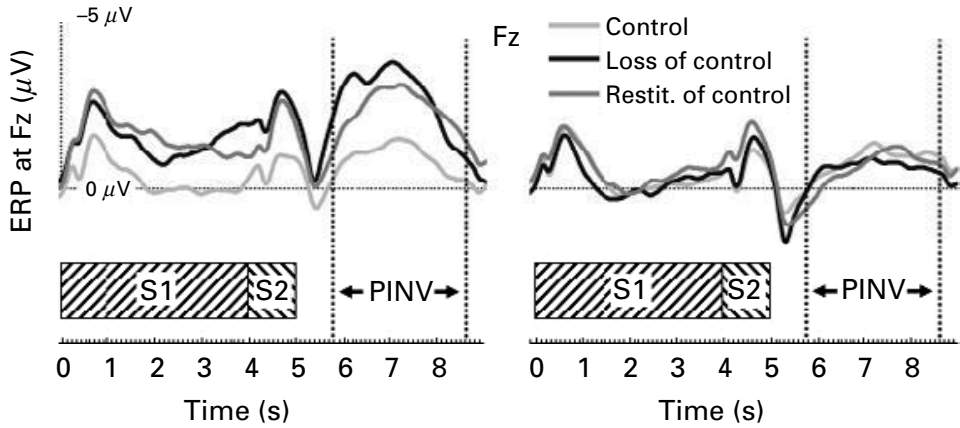

(b)
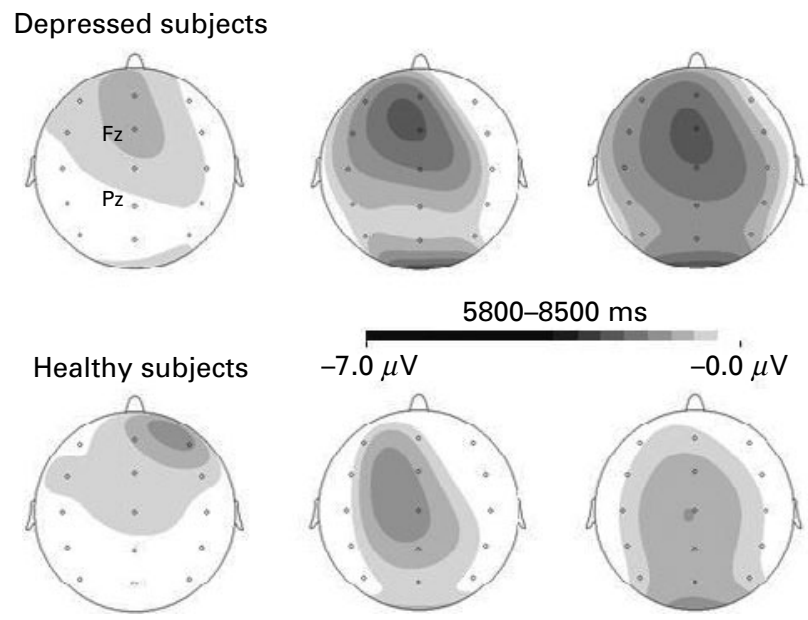

Loss of control

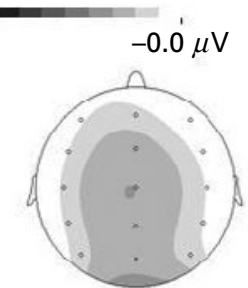

Control

Restitution of control

Fig. 2. (a) Averaged event-related potentials (ERPs, negativity up, filtered with a $6 \mathrm{~Hz}, 12 \mathrm{~dB}$ high cut-off) for the midline frontal recording site (Fz) during S1 (warning stimulus) and S2 (imperative stimulus) presentation, and the post-S2 interval. The post-imperative negative variation [PINV $(\mu \mathrm{V})$ ] was parameterized $800-3500 \mathrm{~ms}$ following $\mathrm{S} 2$ termination. The lines indicate the ERPs during initial condition of control, subsequent loss of control and restitution of control for depressed $(n=26)$ and healthy $(n=24)$ subjects. (b) Topographic two-dimensional maps of the post-S2 interval (800-3500 ms) for depressed and healthy subjects during initial condition of control, subsequent loss of control and restitution of control [topographic interpolation by spherical splines (order $=4$, maximum degree of Legendre polynomials $=10, \lambda=1 \times \mathrm{e}^{-5}$ ), see Perrin et al. 1989]. Pz, parietal recording site.

To control for a possible influence of perceived helplessness on the identified main and interaction effects at $\mathrm{Fz}$, we reanalysed the data with baseline helplessness as a covariate. The reported group and interaction effects remained statistically significant [group $F(1,47)=4.37, p=0.042$; condition $\times$ group $F(2,46)=8.03, p=0.001]$.

To further control for a differential effect of the electrical stimulation on PINV magnitudes, we included the number of electrical stimuli during control and during restitution of control as covariates in the ANOVA. Again, this procedure did not affect the significance of our results [group: $F(1,46)=6.39, p=$ 0.015 ; condition: $F(2,45)=3.97, p=0.026$; condition $\times$ group: $F(2,45)=8.83, p=0.001]$. Averaged eventrelated potentials for $\mathrm{Fz}$ are illustrated in Fig. $2 a$.

\section{Parietal recording site}

The ANOVA for $\mathrm{Pz}$ as a control site resulted in no significant effects [group: $F(1,48)=1.28$, N.S.; condition: $\quad F(2,47)=2.53$, N.S.; condition $\times$ group: $F(2,47)=0.424$, N.s.]. Fig. $2 b$ shows the topographic distribution of the PINV during the post-imperative interval.

\section{Exclusion of patients with dysthymia}

To test for potential confounds arising from the inclusion of mildly chronically depressed patients, we reanalysed the data by excluding individuals with pure dysthymia $(n=7)$. The results regarding subjective ratings, errors and PINV magnitudes remained virtually the same [e.g. PINV at Fz, 


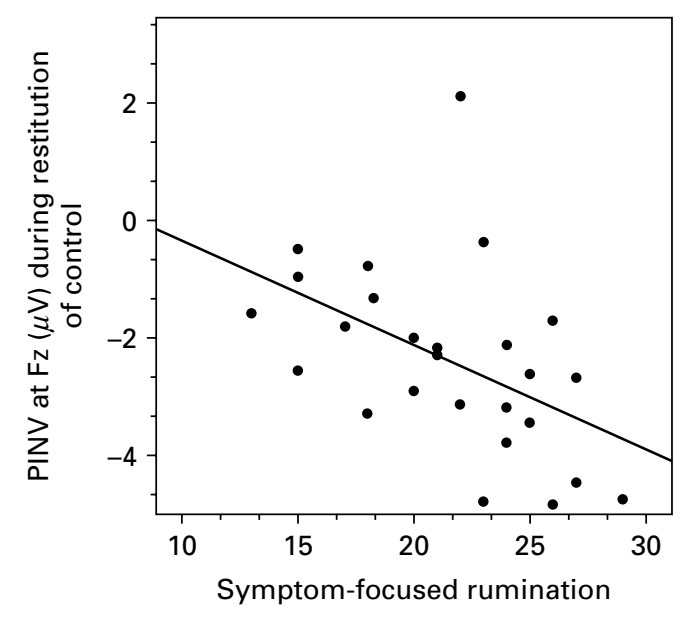

Fig. 3. Scatterplot for frontal post-imperative negative variation (PINV) magnitudes $(\mu \mathrm{V})$ at the midline frontal recording site $(\mathrm{Fz})$ during restitution of control and symptom-focused rumination scores measured by the German version of the response styles questionnaire (Kuehner et al. 2007).

group: $F(1,41)=7.18, p=0.011$; condition: $F(2,40)=$ $3.11, p=0.056$; condition $\times$ group: $F(2,40)=8.55, p<$ 0.001].

\section{Correlations between frontal PINV and measures of depression and cognitive vulnerability}

In the total sample, higher levels of self-(BDI-II) and interviewer-(HAMD) rated depression were connected to larger (more negative) frontal PINV magnitudes during loss of control (BDI-II: partial $r=-0.363$, $p<0.05$; HAMD: partial $r=-0.492, p<0.001)$ and restitution of control (BDI-II: partial $r=-0.489$, $p<0.001$; HAMD: partial $r=-0.491, p<0.001)$. However, these results merely mirrored respective group differences identified in the ANOVAs, since separate correlation analyses for the depressed and healthy subsamples resulted in no significant correlations within groups (all $p>0.10$ ). Perceived helplessness was not significantly correlated with PINV values in any condition. Particularly in the depressed subsample, we identified significant negative correlations between the two rumination scores and PINV magnitude under restitution of control. In this group, higher levels of symptom-focused and self-focused rumination were connected to larger PINV values under restitution of control, even if baseline depression levels were partialled out $(n=26, \mathrm{PINV}$ at Fz during restitution of control with symptom-focused rumination: partial $r=-0.517, p=0.01$, with selffocused rumination: partial $r=-0.419, p=0.05)$. In contrast, respective coefficients failed to reach statistical significance in the healthy subsample (all $p>0.10$ ). Fig. 3 presents a scatterplot for the association of symptom-focused rumination and frontal PINV magnitudes during restitution of control in depressed patients.

\section{Discussion}

This study investigated cognitive, behavioural and physiological effects of stressor uncontrollability in depressed individuals and healthy controls. While previous studies have successfully manipulated stressor controllability by means of change from a condition of control to loss of control in forewarned reaction (S1-S2) paradigms (Rockstroh et al. 1979), we expanded the standard procedure by a subsequent condition of restitution of control to also assess the effects of previous stressor uncontrollability in a situation where control was objectively re-established. Additionally, we included ratings of arousal, emotional valence, controllability and helplessness throughout the experiment.

While depressed participants rated the situation as more unpleasant in general, ratings of emotional valence did not vary with the alternating pattern of controllability. Both groups demonstrated only a slight increase of errors after withdrawal of control. This indicates that the challenge of the task was moderate and comparable for both healthy and depressed individuals.

Both groups rated their perceived controllability in concordance with the varying degrees of controllability. However, depressed participants felt more helpless and aroused throughout the experiment. While withdrawal of control caused a non-significant increase of perceived helplessness in both groups, only depressed subjects displayed markedly enhanced levels of helplessness in the subsequent restitution of control condition.

The most apparent differences between depressed and healthy subjects were identified with respect to frontal PINV magnitudes. During loss of control, depressed participants showed an enhanced PINV over frontal sites, indicating that they engaged frontal areas to resolve task-induced ambiguity (see Klein et al. 1996). Furthermore, depressed participants maintained this activation pattern in the subsequent condition when control was re-established. They also felt more helpless than healthy controls, particularly under restitution of control. Thus, while the enhanced frontal PINV in depressed subjects during loss of control may be related to heightened contingency reappraisal and task-induced ambiguity, the experience of uncontrollability appeared to have also biased subsequent cortical processing, as expected by learned helplessness theory. Since this was only true for the depressed subsample, we assume that depressed 
individuals are more susceptible to conditions of uncontrollability.

The event-related potential data indicate that depressed individuals responded to changing levels of stressor controllability with enhanced prefrontal activation. Increased prefrontal activity during loss of control and restitution of control may reflect a compensatory mechanism in order to resolve taskinduced ambiguity. These considerations are in line with recent studies indicating abnormal prefrontal hyperactivation during working memory load in patients with mood disorders (Hugdahl et al. 2004; Harvey et al. 2005; Rose et al. 2006; Wagner et al. 2006; Chiu \& Deldin, 2007). It is thus conceivable that depressed subjects need enhanced activation of prefrontal brain regions such as the anterior cingulate cortex, the ventromedial or the dorsolateral prefrontal cortex (Luu et al. 2003; Rose et al. 2006; Siegle et al. 2007), which are involved in cognitive control during ambiguity provoked by uncontrollable stress. In this context, future studies using functional magnetic resonance imaging (fMRI) to identify specific neuroanatomical areas involved in the observed altered frontal response pattern in depressed patients are warranted.

Finally, habitual symptom-focused and selffocused rumination were specifically linked to the frontal PINV under restitution of control, and this was particularly true for the depressed subsample. These results are the first to suggest a possible connection between psychological traits reflecting perpetual preoccupation with negative aspects of the self and altered prefrontal information processing during a learned helplessness paradigm in depressed patients.

This study has several limitations. First, the PINV cannot be regarded as a depression-specific cortical index of altered information processing. An enhanced PINV has also been observed in schizophrenic patients where it is similarly regarded as a cortical indicator of task-related ambiguity (Klein et al. 1996; Verleger et al. 1999). Second, our sample size was probably not large enough to identify higher-order interactions (e.g. between group, condition and electrode site) with sufficient statistical power. Finally, the cross-sectional design of our study does not allow us to decide whether the observed enhanced frontal PINV in depressed patients may be regarded as a state or a trait marker of altered information processing. To clarify this issue, a longitudinal study that also compares clinically remitted and non-remitted depressed patients is in progress.

In conclusion, the present findings indicate that non-medicated depressed individuals are more susceptible to conditions of stressor uncontrollability.
While the PINV has been established as a reliable indicator of loss of control in previous work (Rockstroh et al. 1979; Bolz \& Giedke, 1981; Lutzenberger et al. 1981; Elbert et al. 1982; Kathmann et al. 1990), this is the first study to show that in depressed subjects the experience of uncontrollability appears to induce biased subsequent cortical processing. During restitution of control, depressed participants still demonstrated elevated frontal PINV magnitudes and pronounced feelings of helplessness. Our data suggest that the prefrontal hyperactivation may be considered as a physiological compensation to resolve ambiguity induced by previous loss of control. Furthermore, we identified substantial associations between enhanced frontal PINV magnitudes with habitual rumination in depressed patients.

Future studies should examine state- versus traitcharacteristics of the PINV, its predictive role for the development and clinical course of depression, and its sensitivity to therapeutic change. Furthermore, studies including a simultaneous assessment of EEG and fMRI are needed to link exaggerations of the PINV in depressed patients to functional neuroanatomical models of depression.

\section{Acknowledgements}

This work was supported by the Deutsche Forschungsgemeinschaft (SFB636/D4). We gratefully acknowledge the valuable assistance of Elena Maininguer in data collection.

\section{Declaration of Interest}

None.

\section{References}

Alfonso J, Frasch AC, Flugge G (2005). Chronic stress, depression and antidepressants: effects on gene transcription in the hippocampus. Annual Review of Neuroscience 16, 43-56.

APA (1994). Diagnostic and Statistical Manual of Mental Disorders, 4th edn (DSM-IV). American Psychiatric Press: Washington, DC.

Beck AT, Steer RA, Brown GK (1996). Beck Depression Inventory, 2nd edn. The Psychological Corporation: San Antonio, TX.

Bolz J, Giedke H (1981). Controllability of an aversive stimulus in depressed patients and health controls: a study using slow brain potentials. Biological Psychiatry 16, 441-452.

Breier A (1989). Experimental approaches to human stress research: assessment of neurobiological mechanisms of stress in volunteers and psychiatric patients. Biological Psychiatry 26, 438-462. 
Chiu PH, Deldin PJ (2007). Neural evidence for enhanced error detection in major depressive disorder. American Journal of Psychiatry 164, 608-616.

Czéh B, Michaelis T, Watanabe T, Frahm J, de Biurrun G, van Kampen M, Bartolomucci A, Fuchs E (2001). Stressinduced changes in cerebral metabolites, hippocampal volume, and cell proliferation are prevented by antidepressant treatment with tianeptine. Proceedings of the National Academy of Sciences USA 98, 12796-12801.

Elbert T, Rockstroh B, Lutzenberger W, Birbaumer N (1982). Slow brain potentials after withdrawal of control. Archiv für Psychiatrie und Nervenkrankheiten 232, 201-214.

First MB, Spitzer RL, Gibbon M, Williams JBW (1997). Structured Clinical Interview for DSM-IV Axis I Disorders - Clinical Version. American Psychiatric Press: Washington, DC.

Gratton G, Coles MG, Donchin E (1983). A new method for off-line removal of ocular artifacts. Electroencephalography and Clinical Neurophysiology 55, 468-484.

Hamilton M (1960). Development of a rating scale for depression. Journal of Neurology, Neurosurgery and Psychiatry 23, 56-62.

Harvey PO, Fossati P, Pochon JB, Levy R, Lebastard G, Lehericy S, Allilaire JF, Dubois B (2005). Cognitive control and brain resources in major depression: an fMRI study using the $n$-back task. Neuroimage 26, 860-869.

Henn FA, Vollmayr B (2005). Stress models of depression: forming genetically vulnerable strains. Neuroscience and Biobehavioral Reviews 29, 799-804.

Hugdahl K, Rund BR, Lund A, Asbjørnsen A, Egeland J, Ersland L, Landro NI, Roness A, Stordal KI, Sundet K, Thomsen T (2004). Brain activation measured with fMRI during a mental arithmetic task in schizophrenia and major depression. American Journal of Psychiatry 161, 286-293.

Kathmann N, Jonitz L, Engel RR (1990). Cognitive determinants of the postimperative negative variation. Psychophysiology 27, 256-263.

Klein C, Rockstroh B, Cohen R, Berg P, Dressel M (1996). The impact of performance uncertainty on the postimperative negative variation. Psychophysiology 33, 426-433.

Kuehner C (2003). Gender differences in unipolar depression: an update of epidemiological findings and possible explanations. Acta Psychiatrica Scandinavica 108, 163-174.

Kuehner C, Holzhauer S, Huffziger S (2007a). Decreased cortisol response to awakening is associated with cognitive vulnerability to depression in a sample of nonclinical young adults. Psychoneuroendocrinology 32, 199-209.

Kuehner C, Huffziger S, Nolen-Hoeksema S (2007b). German Version of the Response Styles Questionnaire (RSQ-D). Manual. Hogrefe: Göttingen, Germany.

Lang P (1980). Behavioral treatment and bio-behavioral assessment: computer applications. In Technology in Mental Health Care Delivery Systems (ed. J. B. Sidowski, J. H. Johnson and T. Williams), pp. 119-137. Ablex: Norwood, NJ.
Lutzenberger W, Elbert T, Rockstroh B, Birbaumer N, Stegagno L (1981). Slow cortical potentials in subjects with high or low scores on questionnaire measuring physical anhedonia and body image distortion. Psychophysiology 18, 371-380.

Luu P, Tucker DM, Derryberry D, Reed M, Poulsen C (2003). Electrophysiological responses to errors and feedback in the process of action regulation. Psychological Science 14, 47-53.

Maier SF (1984). Learned helplessness and animal models of depression. Progress in Neuro-psychopharmacology and Biological Psychiatry 8, 435-446.

Maier SF, Seligman ME (1976). Learned helplessness and depression. Journal of Experimental Psychology 105, 3-46.

Matsuo K, Glahn DC, Peluso MA, Hatch JP, Monkul ES, Najt P, Sanches M, Zamarripa F, Li J, Lancaster JL, Fox PT, Gao JH, Soares JC (2007). Prefrontal hyperactivation during working memory task in untreated individuals with major depressive disorder. Molecular Psychiatry 12, 158-166.

Mayberg HS (1997). Limbic-cortical dysregulation: a proposed model of depression. Journal of Neuropsychiatry and Clinical Neurosciences 9, 471-481.

Nolen-Hoeksema S (2004). The response styles theory. In Depressive Rumination: Nature, Theory, and Treatment of Negative Thinking in Depression (ed. C. Papageorgiou and A. Wells), pp. 107-123. Wiley: New York.

Nolen-Hoeksema S, Morrow J (1991). A prospective study of depression and posttraumatic stress symptoms after a natural disaster: the 1989 Loma Prieta earthquake. Journal of Personality and Social Psychology 61, 115-121.

Nuwer MR, Comi G, Emerson R, Fuglsang-Frederiksen A, Guerit JM, Hinrichs H, Ikeda A, Luccas FJ,

Rappelsberger P (1998). IFCN standards for digital recording of clinical EEG. International Federation of Clinical Neurophysiology. Electroencephalography and Clinical Neurophysiology 106, 259-261.

Perrin F, Pernier J, Bertrand O, Echallier JF (1989). Spherical splines for scalp potential and current density mapping. Electroencephalography and Clinical Neurophysiology 72, 184-187.

Picton TW, Lins O, Scherg M (1995). The recording and analysis of event-related potentials. In Handbook of Neuropsychology, vol. 10 (ed. J. Boller and J. Grafman), pp. 29-99. Elsevier: Amsterdam.

Pizzagalli DA, Sherwood RJ, Henriques JB, Davidson RJ (2005). Frontal brain asymmetry and reward responsiveness: a source-localization study. Psychological Science 16, 805-813.

Rockstroh B, Elbert T, Lutzenberger W, Birbaumer N (1979). Slow cortical potentials under conditions of uncontrollability. Psychophysiology 16, 374-380.

Rose EJ, Simonotto EE, Ebmeier KP (2006). Limbic overactivity in depression during preserved performance on the $n$-back task. Neuroimage 29, 203-215.

Siegle GJ, Thompson W, Carter CS, Steinhauer SR, Thase ME (2007). Increased amygdala and decreased dorsolateral 
prefrontal BOLD responses in unipolar depression: related and independent features. Biological Psychiatry 61, 198-209.

Verleger R, Wascher E, Arolt V, Daase C, Strohm A, Kompf D (1999). Slow EEG potentials (contingent negative variation and post-imperative negative variation) in schizophrenia: their association to the present state and to Parkinsonian medication effects. Clinical Neurophysiology 110, 1175-1192.

Wagner G, Sinsel E, Sobanski T, Köhler S, Marinou V, Mentzel HJ, Sauer H, Schlösser RG (2006). Cortical inefficiency in patients with unipolar depression: an event-related FMRI study with the Stroop task. Biological Psychiatry 59, 958-965. 